\begin{tabular}{|c|c|}
\hline Title & Electromagnetic absorbing materials using nonwoven fabrics coated with multi-walled carbon nanotubes \\
\hline Author(s) & Sano, Eiichi; A kiba, Eiji \\
\hline Citation & $\begin{array}{l}\text { Carbon, } 78,463.468 \\
\text { https://doi.org/10.1016/.carbon.2014.07.027 }\end{array}$ \\
\hline Issue Date & 201411 \\
\hline Doc URL & http:/hdl. handle.net/2115/57441 \\
\hline Type & article (author version) \\
\hline File Information & final manuscript_CARBON78_463.pdf \\
\hline
\end{tabular}

Instructions for use 


\title{
Electromagnetic absorbing materials using nonwoven fabrics coated with multi-walled carbon nanotubes
}

\author{
Eiichi Sano ${ }^{1, *}$ and Eiji Akiba ${ }^{2}$ \\ ${ }^{1}$ Research Center for Integrated Quantum Electronics, Hokkaido University, Sapporo 060-8628, Japan \\ ${ }^{2}$ Research \& Development Department, Kuraray Living Co. Ltd., Osaka 530-8611, Japan
}

\begin{abstract}
We calculated the complex permittivity of materials required to achieve a single-layer electromagnetic absorber with a high absorption coefficient in the $60-\mathrm{GHz}$ band. The calculation results indicated that an absorption coefficient of 0.95 can be achieved for a 5-mm sheet when the real part of the relative permittivity $\varepsilon_{r}$ ' is close to that of the free space and the conductivity $\sigma$ is around $3 \mathrm{~S} / \mathrm{m}$. On the basis of the findings, we developed three types of nonwoven fabrics with high porosity in which the fibers were coated with multi-walled carbon nanotubes (MWCNTs). The $\varepsilon_{r}$ ' of $1.12-1.32$ and $\sigma$ of 0.107-12.4 S/m were obtained depending on the porosity of the fabrics. The measured absorption coefficients reached 0.97 at $60 \mathrm{GHz}$ in agreement with the calculations. The present MWCNTs-coated nonwoven fabrics will be useful materials for high performance electromagnetic absorbers.
\end{abstract}

\footnotetext{
Corresponding author: FAX: +81 11716 6004. E-mail address: esano@rciqe.hokudai.ac.jp (E.Sano).
} 


\section{Introduction}

Wireless equipment has become indispensable in our everyday lives. However, as wireless equipment has become more convenient, electromagnetic interference (EMI) problems have become more serious. To convert electromagnetic (EM) energy into heat and to absorb EM wave, many types of EM absorbers have been developed since the inventions by Jaumann in 1943 and Salisbury in 1952. EM absorbers with multiple layers tend to be thick and to have narrow operation bandwidth. Their performance depends on the combination of the thickness and permittivity (and/or permeability) of each layer. A sophisticated optimization technique such as a genetic algorism (GA) is needed to widen the bandwidth [1]. To thin the absorbers, a frequency selective surface (FSS) [2] and a metamaterial surface [3] have been introduced. In these types of absorbers, the operating bandwidth is determined by the surface frequency characteristics. Nevertheless, a complicated design for the metallic pattern of the FSS using GA is needed to achieve a target performance [2]. The crowded bands of 2.5 and 5 $\mathrm{GHz}$ will push the operation band of wireless communication systems to the millimeter (MM)-wave region (ex. $60 \mathrm{GHz}$ ), in which the anti-collision radar systems for vehicles have also been developed. Since most magnetic materials do not work in the MM-wave region due to their resonance frequency limits, the dielectric loss property must be used for absorbing the MM-wave. The reduced wavelength in the MM-wave region enables absorbers to be thin and opens a way to fabricate single-layer absorbers.

Carbon nanotubes (CNTs) have been attracting much attention due to their excellent electrical, mechanical, and thermal properties [4]. Among these properties, the extremely high conductivity and large aspect ratio are very effective for a filler in conductive composite materials. A lot of work has been done to achieve wide ranges of 
permittivity and conductivity [5-11]. A high conductivity of more than $10^{3} \mathrm{~S} / \mathrm{m}$ has been reported for both single-walled (SW) [12] and multi-walled (MW) [13] CNT composites, while a large real permittivity of 200 at $18 \mathrm{GHz}$ has been observed for MWCNT/cellulose composite paper [14]. These characteristics of CNT composites have been verified to be useful for EMI shielding [13, 14]. Since the high EMI shielding performances of these composites rely on high reflection, however, the reflected waves pose other problems to the generation source itself as the electric noise. A highly absorbing material is desirable for solving EMI problems. As mentioned above, multilayer EM absorbers are complicated to design, fabricate and dispose of. In this paper, we clarify the complex permittivity of the materials required to achieve a single-layer absorber with a high absorption coefficient in the MM-wave region. On the basis of the findings, we develop nonwoven fabrics coated with MWCNTs appropriate to achieve an efficient absorber.

\section{Experimental details}

\subsection{Material design}

By taking into account the multiple reflections for the three-layer system shown in Fig. 1(a), the coefficients of transmission $T$, reflection $R$, and absorption $A$ are given by

$$
T=t t^{*}, R=r r^{*}, A=1-T-R,
$$

where

$$
t=\frac{t_{12} t_{23} \exp (-j \Delta)}{1+r_{12} r_{23} \exp (-2 j \Delta)}
$$

and

$$
r=\frac{r_{12}+r_{23} \exp (-2 j \Delta)}{1+r_{12} r_{23} \exp (-2 j \Delta)}
$$


with

$$
\Delta=k_{0} n_{2} d \cos \theta_{r} .
$$

Here, $t^{*}$ and $r^{*}$ are respectively the complex conjugates of $t$ and $r, r_{m n}$ and $t_{m n}$ are respectively the reflection and transmission ratios of the complex electric field amplitudes (from medium $m$ to $n$ ), $k_{0}$ is the wave number in the free space, $n_{2}$ and $d$ are respectively the complex index of refraction and the thickness of Medium 2, $\theta_{r}$ is the refraction angle calculated by Snell's law, and $j=\sqrt{-1}$. The ratios $r_{m n}$ and $t_{m n}$ for perpendicular polarization are given by

$$
r_{m n}=\frac{\eta_{n} \cos \theta_{i}-\eta_{m} \cos \theta_{r}}{\eta_{n} \cos \theta_{i}+\eta_{m} \cos \theta_{r}} \text { and } t_{m n}=r_{m n}+1
$$

with the wave impedances $\eta_{m}$ and $\eta_{n}$ of Medium $m$ and $n$ [15]. We examined a single absorber by treating Media 1 and 3 as air. The permeability of Medium 2 was assumed to be equal to that of vacuum, while the complex permittivity in Medium 2 was expressed as

$$
\varepsilon_{2}=\varepsilon_{0}\left(\varepsilon_{r}^{\prime}-j \varepsilon_{r}^{\prime \prime}\right)=\varepsilon_{0}\left(\varepsilon_{r}^{\prime}-j \frac{\sigma}{\omega \varepsilon_{0}}\right) .
$$

Here, $\varepsilon_{0}$ is the permittivity of vacuum, $\sigma$ is the conductivity, and $\omega$ is the angular frequency.

Fig. 1(b) shows a contour plot of the calculated absorption coefficient in the $\varepsilon_{r}{ }^{\prime}-\sigma$ plane for the normal incident $\left(\theta_{i}=0\right)$ and $d=5 \mathrm{~mm}$ at $60 \mathrm{GHz}$. The calculation result indicated that an absorption coefficient of 0.95 can be achieved with only a single layer when $\varepsilon_{r}$ ' is close to that of the free space and $\sigma$ is around $3 \mathrm{~S} / \mathrm{m}$. In Fig. $1(\mathrm{~b})$, the values of $\varepsilon_{r}$ ' and $\sigma$ reported for the CNT composites [5-11] are plotted, although they were not measured at $60 \mathrm{GHz}$. (They were measured at $1 \mathrm{GHz}$ in [5], $12 \mathrm{GHz}$ in [8], $18 \mathrm{GHz}$ in $[7,11]$, and $40 \mathrm{GHz}$ in $[6,9,10]$.) The reported CNT composites seemed to be 
unsuitable for the single-layer absorbers with sufficient absorption coefficients, although the reported values of $\varepsilon_{r}$ ' and $\sigma$ might decrease slightly at $60 \mathrm{GHz}$.

(a)

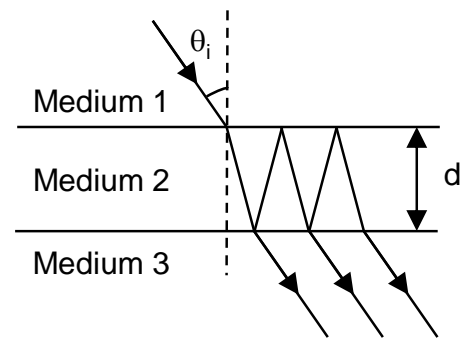

(b)

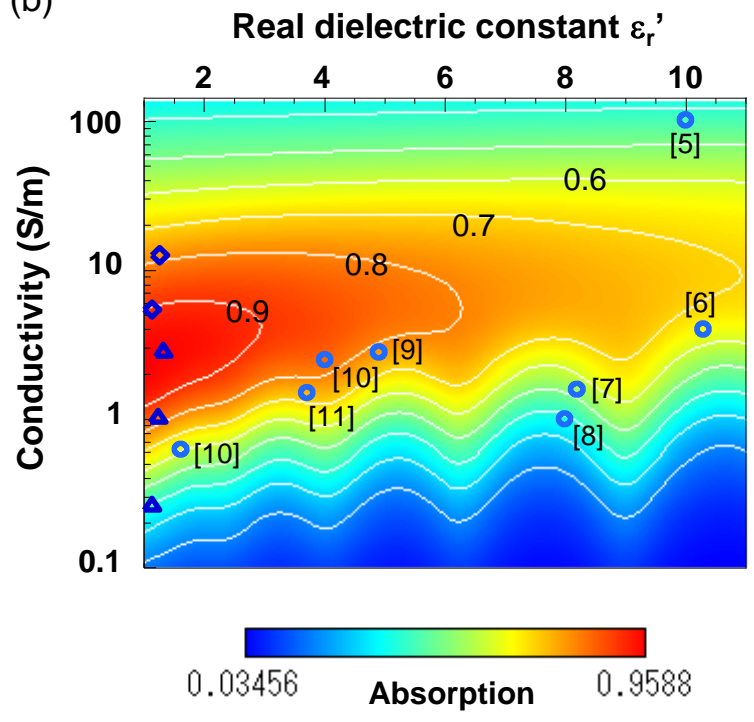

Fig. 1 (a) Cross section of three-layer system and (b) contour plot of calculated absorption coefficient in $\varepsilon_{r}$ ' $\sigma$ plane. Open circles: $\varepsilon_{r}^{\prime}$ and $\sigma$ reported for CNT composites [5-11]. Open triangles and diamonds: $\varepsilon_{r}$ ' and $\sigma$ achieved in this work.

The dielectric constants of CNT composites need to be modeled in order to explore the way to achieve $\varepsilon_{r}$ ' and $\sigma$ values that are suitable for large absorption coefficients. To do this, we used the Maxwell-Garnett model given by [16]

$$
\varepsilon_{e f f}=\varepsilon_{i} \frac{[N+f(1-N)] \varepsilon_{C N T}+(1-N)(1-f) \varepsilon_{i}}{N(1-f) \varepsilon_{C N T}+(f N+1-N) \varepsilon_{i}} .
$$

Here, $\varepsilon_{i}$ is the dielectric constant of a base material, $f$ is the filling factor (CNT content), and $N$ is the geometrical factor expressed as 


$$
N \approx \frac{r^{2}}{a^{2}} \ln \frac{a}{r}
$$

for CNTs with a length of $2 a$ and a radius of $r$. The dielectric constant of CNTs was approximated by the Drude-Lorentz model as in [16]

$$
\varepsilon_{C N T}=\varepsilon^{\infty}-\frac{\omega_{p}^{2}}{\omega^{2}-j \Gamma \omega}+\frac{\omega_{p l}^{2}}{\omega_{l}^{2}-\omega^{2}+j \Gamma_{l} \omega} .
$$

Figs. 2(a) and 2(b) respectively show the contour plots of the real part of $\varepsilon_{e f f}$ and the conductivity [calculated by $\sigma=\operatorname{Im}\left(\varepsilon_{e f f}\right) \varepsilon_{0} \omega$ ], both calculated at $60 \mathrm{GHz}$, in the $\varepsilon_{i}-f$ plane. The parameter values deduced from terahertz time-domain spectroscopy measurements for SWCNTs [16] were used in the calculation. They were: $\varepsilon^{\infty}=3.24$, $\omega_{p} / 2 \pi=5.4 \mathrm{THz}, \Gamma / 2 \pi=1.17 \mathrm{THz}, \omega_{p l} / 2 \pi=6.19 \mathrm{THz}, \omega_{l} / 2 \pi=2.4 \mathrm{THz}$, and $\Gamma_{l} / 2 \pi=4.56$ THz. Although they were deduced for SWCNTs, these parameter values well reproduced the measured permittivity of our sample described in the next section. It is difficult to obtain CNT composites with $\varepsilon_{r}$ ' (the real part of $\varepsilon_{e f f}$ ) close to 1.0 using dense base materials such as silica, ceramic, and even polymer, because their dielectric constants are larger than 2. Fig. 2(b) clearly indicates that precise control of CNT content is needed to achieve $\sigma$ of around $3 \mathrm{~S} / \mathrm{m}$. The dielectric constant $\varepsilon_{i}$ of a porous base material with a porosity of $p$ can be expressed as

$$
\varepsilon_{i}=\varepsilon_{\text {air }} p+\varepsilon_{d}(1-p)
$$

where $\varepsilon_{d}$ is the dielectric constant of solid base material, and $\varepsilon_{a i r}=1$. The filling factor $f$ in Eq. (6) can be approximated by

$$
f=(1-p) v
$$

where $v$ is the volume fraction of CNTs. Fig. 3 shows the calculated dependences of the real and imaginary parts of dielectric constant $\varepsilon_{\text {eff }}$ and the conductivity on porosity $p$ for a CNT composite with $v=3.5 \%$. A low dielectric constant close to 1.0 and a conductivity 
of $3 \mathrm{~S} / \mathrm{m}$ suitable for large absorption are expected to be achieved by using a porous base material with a porosity larger than 0.9 . Using nonwoven fabrics with high porosity as base materials seem to be very effective to achieve such $\varepsilon_{r}$ ' and $\sigma$.
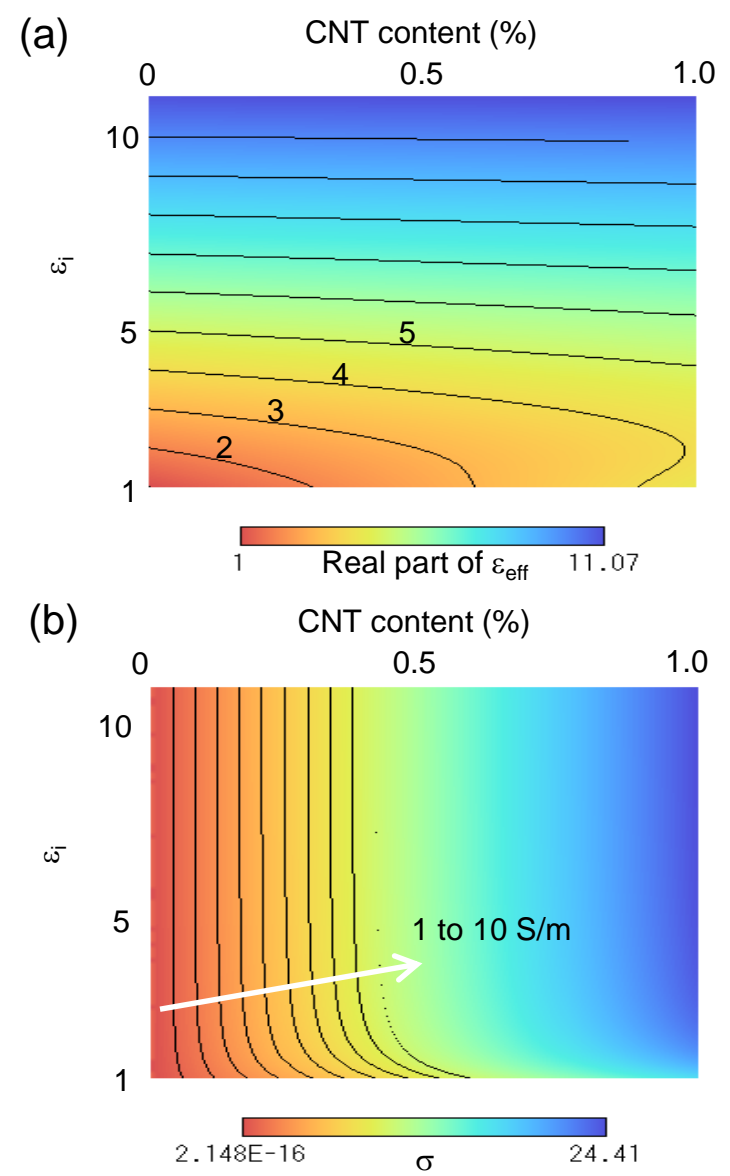

Fig. 2 Contour plots of (a) real part of $\varepsilon_{\text {eff }}$ and (b) conductivity $\left[\sigma=\operatorname{Im}\left(\varepsilon_{\text {eff }}\right) \varepsilon_{0} \omega\right]$. Both were calculated at $60 \mathrm{GHz}$. 


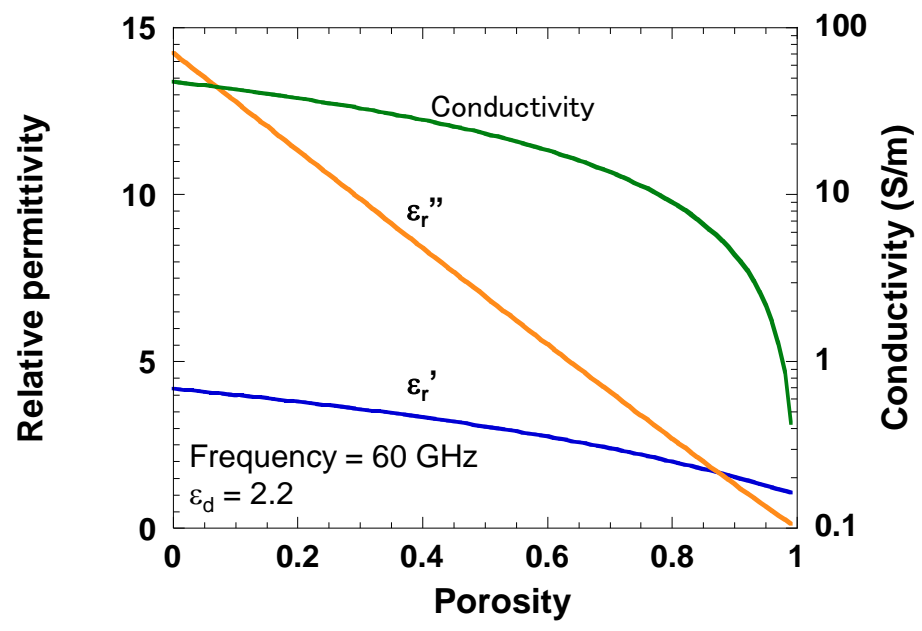

Fig. 3 Calculated dependences of real and imaginary parts of dielectric constant $\varepsilon_{\text {eff }}$ and conductivity on porosity $p$ for a CNT composite with $v=3.5 \%$.

The frequency dependence of the permittivity causes the variation in the absorption coefficient in the operating frequency region. In addition, degradation in the absorption coefficient was a concern when the incident angle $\theta_{i}$ increased. Fig. 4(a) shows the calculated dependences of the coefficients $T, R$, and $A$ on the frequency. The solid lines in Fig. 4(a) were calculated with the Maxwell-Garnett model [frequency-dependent permittivity and conductivity shown in Fig. 4(b)], while the dashed lines were calculated with the frequency-independent $\varepsilon_{r}$ ' of 1.2 and $\sigma$ of $2 \mathrm{~S} / \mathrm{m}$. The frequency dependence of the permittivity had little influence on the coefficients. Fig. 4(c) shows the calculated dependences of $T, R$, and $A$ on the incident angle. The calculation showed that the absorption coefficient was larger than 0.9 for $\theta_{i}<50$ degrees. 

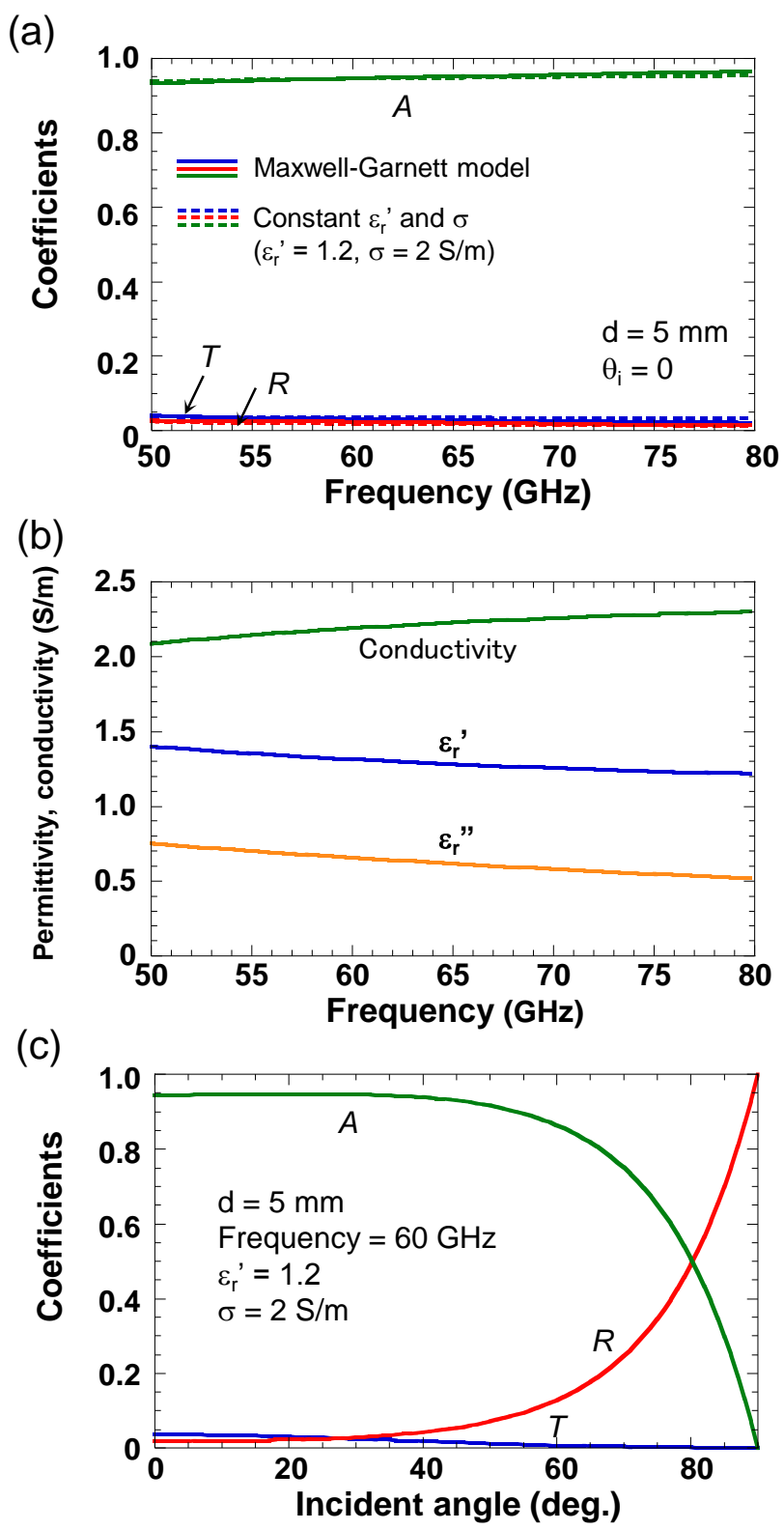

Fig. 4 Calculated dependences of coefficients $T, R$, and $A$ on (a) incident angle and (b) frequency.

\subsection{Fabrication and evaluation methods}

We developed three types of MWCNT-coated nonwoven fabrics. The basic fabrics were as follows: $a$; spunbond-meltblown-spunbond (SMS) type polyester (PET) nonwoven fabric (Asahi-Kasei, CC5020, specific gravity $(\mathrm{SG})=0.2 \mathrm{~g} / \mathrm{cm}^{3}$ ), $b$; PET /ethylene-vinyl 
alcohol copolymer $\left(\mathrm{EVAL}^{\mathrm{TM}}\right.$ ) nonwoven fabric (Kuraray, board-type Flextar $^{\mathrm{TM}}$, $\mathrm{SG}=0.15 \mathrm{~g} / \mathrm{cm}^{3}$ ), and $c$; PET thermally bonded nonwoven fabric (Nishikawa Rose, $\mathrm{SG}=0.093 \mathrm{~g} / \mathrm{cm}^{3}$ ). We prepared MWCNT-water suspension using MWCNTs (Bayer, $\mathrm{C} 150 \mathrm{P})$, dipped the basic fabrics into the water suspension, wringed them by a mangle machine, and dried them by using a hot-air oven at $90{ }^{\circ} \mathrm{C}$ to get MWCNT-coated nonwoven fabrics. Since little care is required for the drying process, a convenient hot-air oven was used. The CNT content was calculated by $C\left(W_{2}-W_{1}\right) / W_{2}$, where $W_{1}$ and $W_{2}$ are the weights of a fabric piece before and after CNT coating, respectively, and $C$ is the weight ratio of CNTs to all the solid contents included in the suspension. The porosity was defined as $\left(V-W_{2} / G\right) / V$, where $V$ is the volume of a fabric piece after CNT coating and $G$ is the specific gravity of the solid fabric piece after CNT coating.

The fabricated absorbers were evaluated using a vector network analyzer (Agilent, E8361C) and materials measurement software (Agilent, 85071E) on the basis of a free space method. Two standard gain horn antennas (Millitech, SGH-15) were used, and the two-port system was calibrated by using the gated reflect line (GRL) calibration method. All the absorbers were $21 \times 21 \mathrm{~cm}$. The distance between the absorbers and the port-2 antenna was fixed to $10 \mathrm{~cm}$, while the distance between the absorbers and the port-1 antenna ranged from 9.5 to $9.9 \mathrm{~cm}$ depending on the absorber thickness. In this measurement setup, the absorber was large enough to avoid any environment effects. After the calibration, the accuracy of the measurement system was confirmed by extracting the permittivity values of air and polyethylene film $\left(\varepsilon_{r}{ }^{\prime} \sim 2.2\right.$ at $\left.60 \mathrm{GHz}\right)$. In addition, a waveguide method was used for some samples to verify the experimental results on the basis of the free space method. A 5-mm-thick V-band waveguide sample 
holder was used in the waveguide method. The complex permittivity values of the absorbers were extracted from the measured scattering parameters on both free space and waveguide methods by using the materials measurement software.

In addition to these measurements in the $\mathrm{V}$ band, a microstrip line (MSL) method was used in the $0.01-10 \mathrm{GHz}$ region to evaluate the EM absorption performance of a thin-film absorber which will be described in the next section.

\section{Results and discussion}

Fig. 5 shows scanning electron microscope (SEM) images of MWCNT-coated nonwoven fabric $c$. These SEM images clearly demonstrate that extremely thin MWCNT networks were formed only on the surface of the fibers and suggest that the porosity was almost unchanged after the MWCNT coating which will be verified in Table I later.

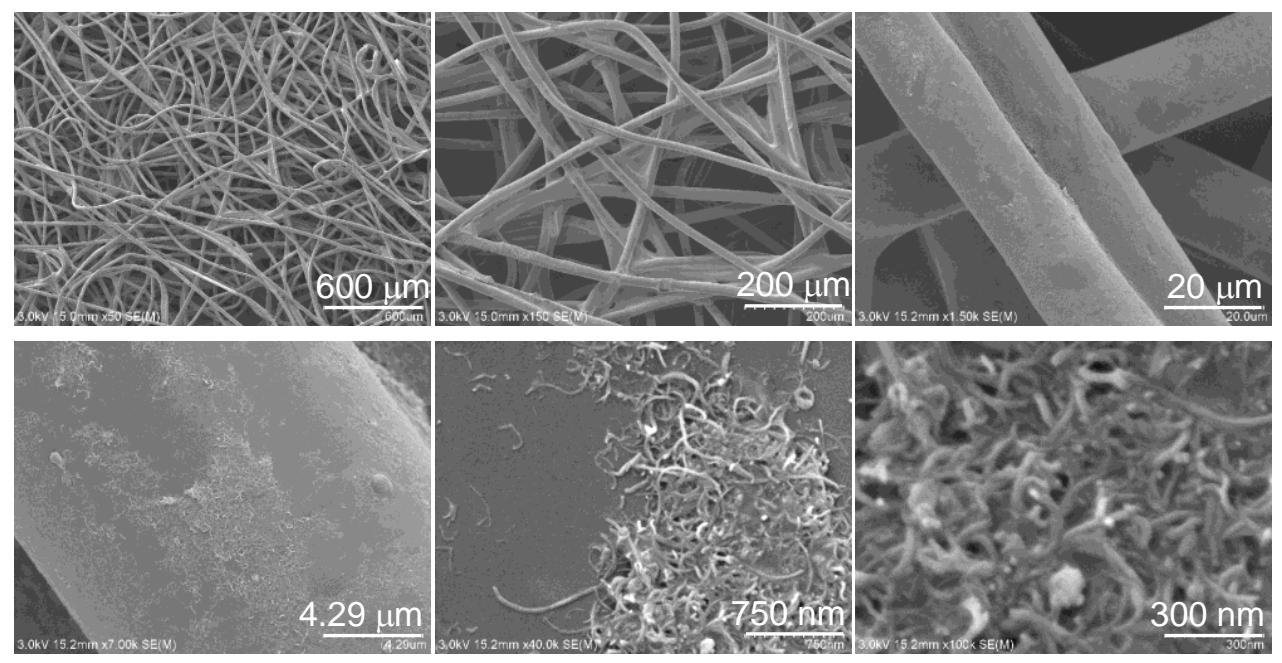

Fig. 5 SEM images of MWCNT-coated nonwoven fabric $c$.

Fig. 6 shows the extracted relative permittivity of fabric $c$ with a CNT content of $3.5 \mathrm{wt} \%$. The relative permittivity values extracted with both methods were almost 
coincident. The solid lines in Fig. 6 were calculated by using Eqs. (6)-(10), where the parameter values in the Drude-Lorentz model were the same as those described above, the volume fraction $v$ of CNTs was assumed to equal $3.5 \mathrm{wt} \%$, and $\varepsilon_{d}=2.2$. Although the parameter values used in the Drude-Lorentz model were deduced from a terahertz time-domain spectroscopy measurements for SWCNTs [16], the calculation well reproduced the measured permittivity of our sample. In Fig. 6, the dashed line was calculated by Eq. (5) with a frequency-independent $\sigma$ of $2.87 \mathrm{~S} / \mathrm{m}$ determined by using $\sigma=\varepsilon_{0} \varepsilon_{r} " \omega$ at $60 \mathrm{GHz}$. A good agreement was obtained between measured and calculated imaginary parts of the permittivity. In the following, the conductance values were calculated using this equation from the imaginary parts of the extracted permittivity. Fig. 7 shows the reflection, transmission, and absorption coefficients of fabric $c$ with a CNT content of $3.5 \mathrm{wt} \%$ measured with the waveguide method. An absorption coefficient of 0.97 was achieved at $60 \mathrm{GHz}$, which agreed well with the calculated value of 0.95 . The calculated reflection and transmission coefficients were respectively 0.04 and 0.01 at 60 $\mathrm{GHz}$, slightly larger than the measured coefficients. The difference between the calculated and measured coefficients might be due to the measurement accuracy. 


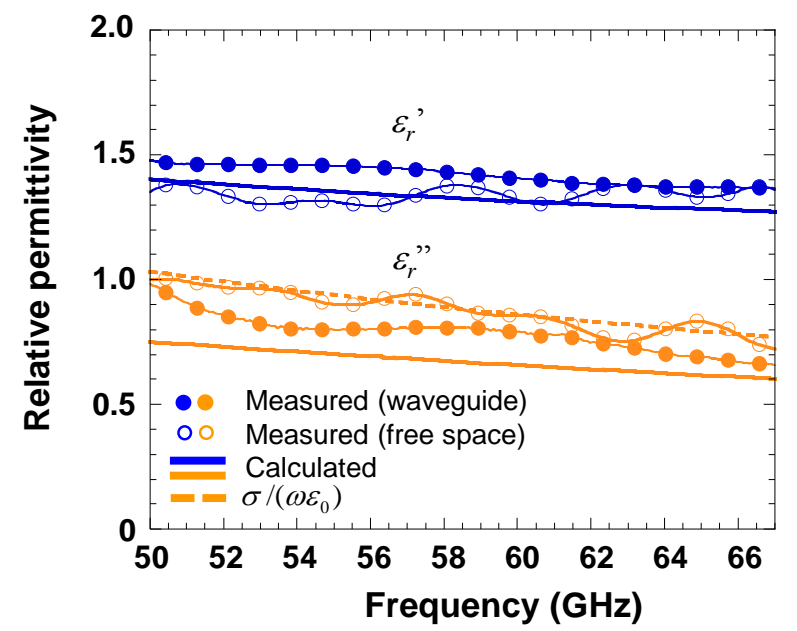

Fig. 6 Relative permittivity of fabric $c$ with a CNT content of $3.5 \mathrm{wt} \%$. Solid lines are the relative permittivity calculated by using the Maxwell-Garnett model. Dashed line shows $\varepsilon_{r}$ " calculated by Eq. (5) with $\sigma$ of $2.87 \mathrm{~S} / \mathrm{m}$.

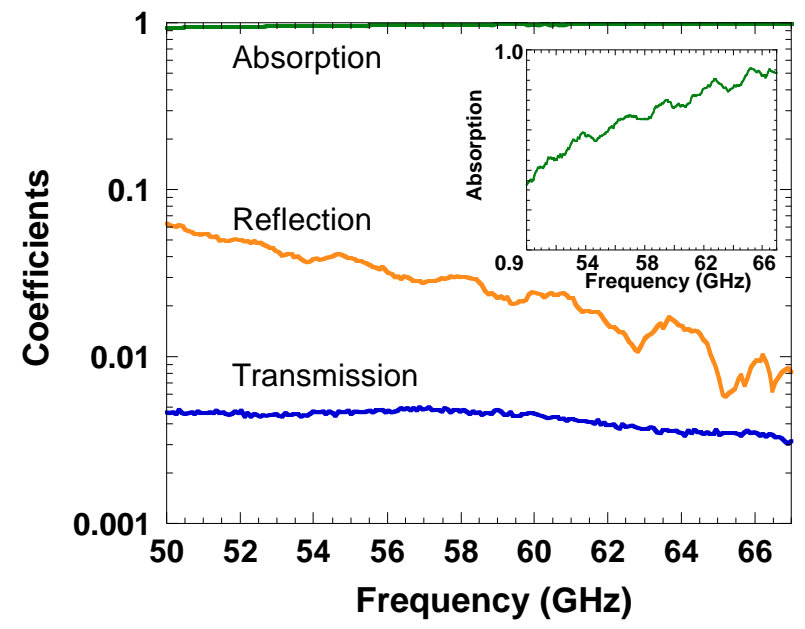

Fig. 7 Reflection, transmission, and absorption coefficients of fabric $c$ with CNT content of $3.5 \mathrm{wt} \%$ measured using waveguide method. Inset shows enlargement of absorption coefficient. 
Table I summarizes the measured results using the free space method. The absorption coefficients measured at $60 \mathrm{GHz}$ closely matched the calculated values. Fig. 8 shows the dependences of $\varepsilon_{r}$ ' and $\sigma$ on the CNT content. The dependence of $\sigma$ on the CNT content clearly demonstrated the percolation characteristics, in which the percolation threshold was around $0.5 \mathrm{wt} \%$. The CNT-coated fabric $b$ seemed to show the highest $\varepsilon_{r}$ ' among the fabrics for a given CNT content. This might be caused by the hydrophilic EVAL. Water which has a large dielectric constant, might increase the $\varepsilon_{r}$ ' of the sample when it is adsorbed on the surface of the fibers uncoated with CNTs. By taking this into account, $\varepsilon_{r}$ ' tend to increase as the porosity increased while the CNT content was kept constant (See fabric $a$ with 3.8-wt\% CNTs and fabric $c$ with 3.5-wt\% CNTs). This was due to the fact that the area of the fiber surfaces coated with CNTs increases as the porosity increases. The values of $\varepsilon_{r}$ ' and $\sigma$ obtained for the CNT-coated fabrics $a$ and $c$ are plotted in Fig. 1(b). The MWCNT-coated nonwoven fabrics achieved low $\varepsilon_{r}$ ' and high $\sigma$ at the same time which has never been obtained by conventional polymer/CNT composites. 
Table I. Summary of measured and calculated results

\begin{tabular}{|c|c|c|c|c|c|c|c|c|c|}
\hline \multirow[t]{3}{*}{ Type } & \multirow{3}{*}{$\begin{array}{c}\text { Porosity } \\
\text { (\%) }\end{array}$} & \multirow{3}{*}{$\begin{array}{c}\text { CNT } \\
\text { content } \\
(w t \%)\end{array}$} & \multirow[t]{3}{*}{$\varepsilon_{\mathrm{r}}{ }^{\prime} *$} & \multirow{3}{*}{$\begin{array}{l}\sigma * * \\
(\mathbf{S} / \mathbf{m})\end{array}$} & \multirow{3}{*}{$\begin{array}{c}\text { Thickness } \\
\text { (mm) }\end{array}$} & \multicolumn{2}{|c|}{ Absorption* } & \multirow{3}{*}{$\begin{array}{l}\text { Measured } \\
\text { reflection * }\end{array}$} & \multirow{3}{*}{$\begin{array}{c}\text { Measured } \\
\text { transmission* }\end{array}$} \\
\hline & & & & & & Measured & Calculated & & \\
\hline & & & & & & & & & \\
\hline$a$ & 72 & 6.0 & 1.26 & 12.4 & 1 & 0.83 & 0.79 & 0.12 & 0.05 \\
\hline$a$ & 72 & 3.8 & 1.13 & 5.37 & 1 & 0.66 & 0.66 & 0.13 & 0.21 \\
\hline$b$ & 89 & 0.6 & 1.24 & 0.314 & 5 & 0.35 & 0.41 & 0.07 & 0.58 \\
\hline$b$ & 89 & 0.3 & 1.19 & 0.107 & 5 & 0.12 & 0.17 & 0.05 & 0.83 \\
\hline$c$ & 94 & 3.5 & 1.32 & 2.87 & 5 & 0.89 & 0.95 & 0.1 & 0.01 \\
\hline$c$ & 94 & 2.1 & 1.22 & 1.03 & 5 & 0.78 & 0.82 & 0.06 & 0.16 \\
\hline$c$ & 95 & 0.5 & 1.12 & 0.267 & 5 & 0.38 & 0.38 & 0.09 & 0.53 \\
\hline
\end{tabular}

* measured and calculated at $60 \mathrm{GHz}$

** calculated by using $\sigma=\varepsilon_{0} \varepsilon_{r}$ " $\omega$ at $60 \mathrm{GHz}$

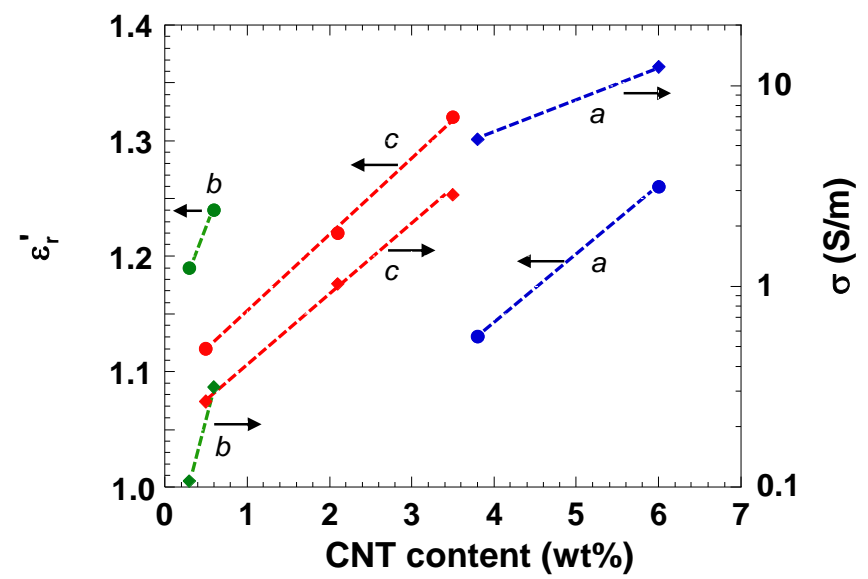

Fig. 8 Dependences of $\varepsilon_{r}{ }^{\prime}$ and $\sigma$ on the CNT content. Dotted lines: eye guide. 
The EM absorption performance of fabric $c$ with a CNT content of $6.0 \mathrm{wt} \%$, a size of $5 \times 5 \mathrm{~cm}$, and a thickness of $0.5 \mathrm{~mm}$ was evaluated by using the MSL method in 0.01-10 GHz region. Fig. 9 shows the measured EM shielding effectiveness (ratio of the absorbed power to the input power). It was almost the same as those of commercially available noise suppression sheets using ferrite materials. Although the measured frequency was restricted to the microwave region due to the loss of the MSL and the SMA connectors mounted on the MSL, the present MWCNT-coated nonwoven fabrics can be used in the MM-wave region, in which ferrite-based materials do not work due to their magnetic properties.

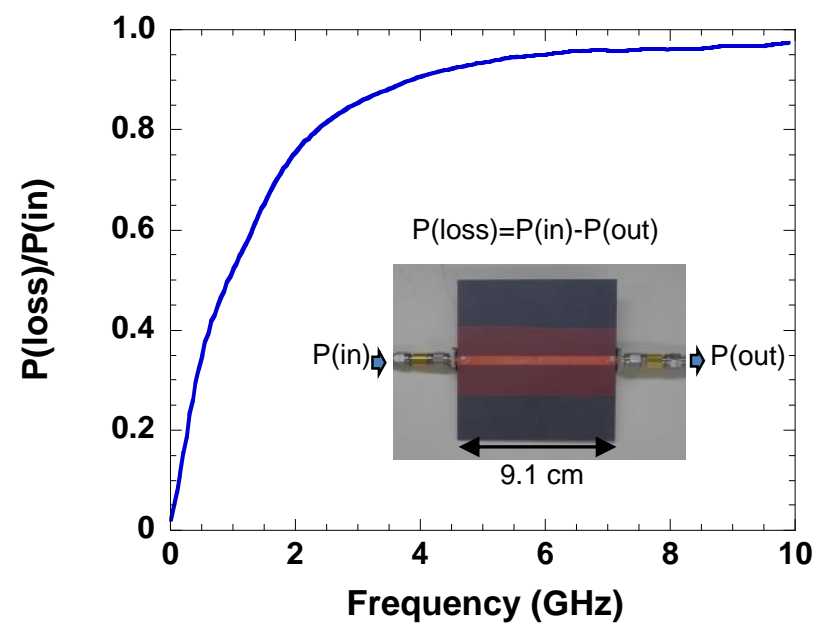

Fig. 9 Measured EM shielding effectiveness. Inset shows photograph of the MSL used in experiments.

\section{Conclusions}

In this work, we clarified the complex permittivity of materials required to achieve a single-layer electromagnetic absorber with a high absorption coefficient in the $60-\mathrm{GHz}$ band. On the basis of the findings, we developed three types of nonwoven fabrics with high porosity in which the fibers were coated with MWCNTs. The MWCNT-coated 
nonwoven fabrics opened up a new region in the $\varepsilon_{r}$ '- $\sigma$ plane. The measured absorption coefficients reached 0.95 in agreement with the calculations. The present MWCNT-coated nonwoven fabrics will be useful materials for high performance electromagnetic absorbers.

\section{Acknowledgements}

The present study was partly supported by a Grant-in-Aid for Scientific Research from the Ministry of Education, Culture, Sports, Science and Technology, Japan. 


\section{REFERENCES}

[1] Matous K, Dvorak GJ. Optimization of electromagnetic absorption in laminated composite plates. IEEE Trans Mag 2003; 39: 1827-35.

[2] Chakravarty S, Mittra R, Williams NR. On the application of the microgenetic algorithm to the design of broad-band microwave absorbers comprising frequency-selective surfaces embedded in multilayered dielectric media. IEEE Trans Microwave Theory Tech 2001; 49: 1050-9.

[3] Zou Y, Jiang L, Wen S, Shu W, Qing Y, Tang Z, Luo H, Fan D. Enhancing and tuning absorption properties of microwave absorbing materials using metamaterials. Appl Phys Lett 2008; 93: 261115-1-3.

[4] Baughman RH, Zakhidov AA, de Heer WA. Carbon nanotubes-the route toward applications. Science 2002; 297: 787-92.

[5] Konyushenko EN, Kazantseva NE, Stejskal J, Trchova M, Kovarova J, Sapurina I, Tomishko MM, Demicheva OV, Prokes J. Ferromagnetic behavior of polyaniline-coated multi-wall carbon nanotubes containing nickel nanoparticles. J Mag Mag Mater 2008; 320: $231-40$.

[6] Xiang C, Pan Y, Guo J. Electromagnetic interference shielding effectiveness of multiwalled carbon nanotube reinforced fused silica composites. Ceramics International 2007; 33: 1293-7.

[7] Zhu H, Lin H, Guo H, Yu L. Microwave absorbing property of Fe-filled carbon nanotubes synthesized by a practical route. Mater Sci Eng B 2007; 138: 101-4.

[8] Xiang C, Pan Y, Liu X, Sun X, Shi X, Guo J. Microwave attenuation of multiwalled carbon nanotube-fused silica composites. Appl Phys Lett 2005; 87: 123103-1-3.

[9] Saib A, Bednarz L, Daussin R, Bailly C, Lou X, Thomassin JM, Pagnoulle C, 
Detrembleur C, Jérôme R, Huynen I. Carbon nanotube composites for broadband microwave absorbing materials. IEEE Trans Microwave Theory Tech 2006; 54: 274554.

[10] Thomassin JM, Pagnoulle C, Bednarz L, Huynen I, Jerome R, Detrembleur C. Foams of polycaprolactone/MWNT nanocomposites for efficient EMI reduction. J Mater Chem 2008; 18: 792-6.

[11] $\mathrm{Wu} \mathrm{J,} \mathrm{Kong} \mathrm{L.} \mathrm{High} \mathrm{microwave} \mathrm{permittivity} \mathrm{of} \mathrm{multiwalled} \mathrm{carbon} \mathrm{nanotube}$ composites. Appl Phys Lett 2004; 84: 4956-8.

[12] Aarab H, Baitoul M, Wery J, Almairac R, Lefrant S, Faulques E, Duvail JL, Hamedoun M. Electrical and optical properties of PPV and single-walled carbon nanotubes composite films. Synth Met 2005; 155: 63-7.

[13] Kim HM, Kim K, Lee CY, Joo J. Electrical conductivity and electromagnetic interference shielding of multiwalled carbon nanotube composites containing $\mathrm{Fe}$ catalyst. Appl Phys Lett 2004; 84: 589-91.

[14] Imai M, Akiyama K, Tanaka T, Sano E. Highly strong and conductive carbon nanotube/cellulose composite paper. Compos Sci Technol 2010; 70: 1564-70.

[15] Pozar DM. Microwave engineering. 2nd ed. NJ: John Wiley \& Sons, Inc.; 1998.

[16] Jeon TI, Kim KJ, Kang C, Maeng IH, Son JH, An KH, Lee JY, Lee YH. Optical and electrical properties of preferentially anisotropic single-walled carbon-nanotube films in terahertz region. J Appl Phys 2004; 95: 5736-40. 